\title{
Fisetin regulates astrocyte migration and proliferation in vitro
}

\author{
NAN WANG, FANG YAO, KE LI, LANLAN ZHANG, GUO YIN, MINGJUN DU and BINGYI WU \\ Research Center of Clinical Medicine, Nanfang Hospital, Southern Medical University, \\ Guangzhou, Guangdong 510515, P.R. China
}

Received September 22, 2015; Accepted January 12, 2017

DOI: 10.3892/ijmm.2017.2890

\begin{abstract}
Fisetin (3,3',4',7-tetrahydroxyflavone) is a plant flavonol found in fruits and vegetables that has been reported to inhibit migration and proliferation in several types of cancer. Reactive astrogliosis involves astrocyte migration and proliferation, and contributes to the formation of glial scars in central nervous system (CNS) disorders. However, the effect of fisetin on the migration and proliferation of astrocytes remains unclear. In this study, we found that fisetin inhibited astrocyte migration in a scratch-wound assay and diminished the phosphorylation of focal adhesion kinase (FAK; Tyr576/577 and paxillin (Tyr118). It also suppressed cell proliferation, as indicated by the decreased number of 5-ethynyl-2'-deoxyuridine (EdU)-positive cells, induced cell cycle arrest in the G1 phase, reduced the percentage of cells in the $\mathrm{G} 2$ and $\mathrm{S}$ phase (as measured by flow cytometry), and decreased cyclin D1 expression, but had no effect on apoptosis. Fisetin also decreased the phosphorylation levels of Akt and extracellular signal-regulated kinase (Erk)1/2, but had no effect on the phosphorylation of p38 mitogenactivated protein kinase (MAPK). These results indicate that fisetin inhibits aggressive cell phenotypes by suppressing cell migration and proliferation via the Akt/Erk signaling pathway. Fisetin may thus have potential for use as a therapeutic strategy targeting reactive astrocytes, which may lead to the inhibition of glial scar formation in vitro.
\end{abstract}

\section{Introduction}

Astrocytes are the most abundant glial subtype in the central nervous system (CNS) and play important roles in interacting with neurons and communicating with other neural cell types (1-4). In spinal cord injury (SCI), astrocytes proximal to lesion zones become reactive. The process of reactive astrogliosis involves the migration and proliferation of astrocytes, contributing to glial scar formation, which is a major impediment to axonal regeneration. Overcoming this physical and biochemical barrier may be crucial for axonal regeneration

Correspondence to: Dr Bingyi Wu, Research Center of Clinical Medicine, Nanfang Hospital, Southern Medical University, 1838 North Guangzhou Avenue, Guangzhou, Guangdong 510515, P.R. China

E-mail: wubingyi1966@sina.com

Key words: fisetin, astrocytes, migration, proliferation and functional compensation during the progression of SCI $(5,6)$. Although extensive investigations have been conducted on SCI (7), no effective therapy has yet been established. The lack of effective interventions is at least partially attributed to the complex process and the failure of axonal regeneration due to glial scaring (7). There is therefore a need to develop therapeutic strategies with which to prevent reactive astrogliosis.

Fisetin $\left(3,3^{\prime}, 4^{\prime}, 7\right.$-tetrahydroxyflavone) is a dietary flavonoid found in fruits and vegetables, including strawberries, persimmons, grapes, cucumbers and onions (8). It has extensive physiological and pharmacological activities, including antioxidant, anti-inflammatory, anti-neoplastic and neuroprotective effects (8-10). It has also been shown that fisetin enhances behavioral performance and attenuates reactive gliosis and inflammation during aluminum chloride-induced neurotoxicity (9). Furthermore, fisetin suppresses oxidative and neuroinflammatory responses in microglial cells (10). However, the effects of fisetin on reactive astrocytes remain unclear. In the present study, we examined whether fisetin inhibits reactive astrogliosis in an in vitro scratch-wound model, and then characterized its underlying mechanisms of action.

\section{Materials and methods}

Materials and reagents. Fisetin (Fig. 1A) (molecular weight, 286.24; Sigma-Aldrich, St. Louis, MO, USA) was used at a purity of $>98 \%$. The molecular structure has been deposited in a publicly accessible database in PubChem with research number: CID 5281614. Stock solutions of fisetin were prepared in dimethyl sulfoxide (DMSO; Sigma-Aldrich) and stored at $-20^{\circ} \mathrm{C}$.

Cell culture. Primary astrocyte cultures were prepared from 1-day-old Sprague-Dawley rat brains (Animal Experiment Center, Southern Medical University, Guangzhou, China), as previously described $(11,12)$. Ethics approval was granted by the Medical Ethics Committee of Southern Medical University, Guangzhou, China. Briefly, a total of 12 rats $(n=3 /$ group) were deeply anesthetized with a mixture of ketamine and xylazine (70:6 mg/kg, intramuscular injection) and then decapitated, the striatums were dissected, and the meninges were removed. The cerebral hemisphere was freed of the meninges and cut into $1 \mathrm{~mm}^{3}$ cubes in Dulbecco's modified Eagle's medium (DMEM; Gibco Life Technologies, Fresno, CA, USA). The tissue was dissociated by vortex mixing for $60 \mathrm{sec}$, and the cell suspension was passed through 70 and $20 \mu \mathrm{m}$ sterile mesh nylon filters. A volume of cell suspension 
containing approximately $4.5 \times 10^{5}$ cells was seeded in a $35 \mathrm{~mm}^{2}$ Falcon tissue culture dish (Corning, Albany, NY, USA). Primary astrocytes were cultured in DMEM containing $10 \%$ fetal bovine serum (FBS) and $0.15 \%$ penicillin-streptomycin reagent (both from Gibco Life Technologies) at $37^{\circ} \mathrm{C}$ with $5 \% \mathrm{CO}_{2}$. The medium was changed twice a week. Cultures of at least 4 weeks were used in the experiments.

Scratch-wound assay. The capacity of the astrocytes to migrate was examined by scratching confluent astrocyte monolayers to induce natural cell migration, as previously reported $(12,13)$. Astrocyte monolayers were wounded by dragging a sterile $200-\mu 1$ pipette tip across the surface. The detached cells and debris were removed immediately with 3 washes of phosphatebuffered saline (PBS). The cells were then maintained for an additional $24 \mathrm{~h}$ in culture medium with $1 \% \mathrm{FBS}$ and various concentrations of fisetin $(25$ and $50 \mu \mathrm{M})$ or $0.1 \%$ DMSO as the control group. Images of the closing wound were acquired under an inverted microscope (Olympus TH4-200; Olympus, Tokyo, Japan) and analyzed using NIS Elements D3.2 (Nikon NT-88-V3; Nikon, Tokyo, Japan).

Cell apoptosis assay. The astrocytes were pre-treated with fisetin $(12.5,25$ and $50 \mu \mathrm{M})$ or $0.1 \%$ DMSO as the control group for $24 \mathrm{~h}$. The cells were then digested with $0.125 \%$ EDTA-free trypsin and collected by centrifugation at 2,000 rpm for $5 \mathrm{~min}$. The cells were washed twice with PBS, resuspended in $500 \mu \mathrm{l}$ binding buffer, and incubated in the dark for $10 \mathrm{~min}$ at room temperature with $5 \mu \mathrm{l}$ of Annexin V-Fluor 488 and $5 \mu \mathrm{l}$ of propidium iodide (PI) (product code: KGAV103; KeyGen, Nanjing, China). The samples were assessed by flow cytometry (BD Biosciences, Mountain View, CA, USA).

Click-iT 5-ethynyl-2'-deoxyuridine (EdU) test. The astrocytes were treated simultaneously with various concentrations of fisetin $(12.5-50 \mu \mathrm{M})$ or $0.1 \%$ DMSO as the control group and EdU $(10 \mu \mathrm{M})$, using the Cell-Light ${ }^{\mathrm{TM}}$ EdU Apollo 567 in vitro flow cytometry kit (product code: C10338-3; Guangzhou RiboBio Co., Ltd., Guangzhou, China) for 24 h. The cells were then stained according to the following protocol: the cells were collected by centrifugation at $1,500 \mathrm{rpm}$ for $5 \mathrm{~min}$, $4 \%$ paraformaldehyde was added at room temperature for 30 min to fix the cells, followed by washing with PBS, then the addition of $0.5 \%$ Triton X-100 for 15 min, 2 washes with PBS, and incubation with click reaction buffer $(100 \mathrm{mM}$ Tris- $\mathrm{HCl}$, pH 8.5; $1 \mathrm{mM} \mathrm{CuSO}_{4} ; 100 \mu \mathrm{M}$ Apollo 550 fluorescent azide; and $100 \mathrm{mM}$ ascorbic acid) for $30 \mathrm{~min}$ in the dark, washing with $0.5 \%$ Triton X-100, and a final addition of $500 \mu \mathrm{l}$ PBS for the flow cytometry analysis, within $1 \mathrm{~h}$.

Cell cycle assay. The astrocytes were synchronized by starved for $12 \mathrm{~h}$ in serum-free medium and treated subsequently with different concentrations of fisetin $(12.5-50 \mu \mathrm{M})$ or $0.1 \%$ DMSO as the control group for $24 \mathrm{~h}$ prior to analysis. Cell cycle analysis was performed by PI staining. The astrocytes were trypsinized and washed with ice-cold PBS (pH 7.4) and fixed in ice-cold $75 \%$ ethanol for $>2 \mathrm{~h}$. The cells were then washed with PBS, treated with $100 \mu \mathrm{l}$ RNase $\mathrm{A}$ at $37^{\circ} \mathrm{C}$ for $30 \mathrm{~min}$, and finally stained with $400 \mu \mathrm{l} \mathrm{PI}$ in the dark at $4^{\circ} \mathrm{C}$ for $30 \mathrm{~min}$. Flow cytometric analysis was then carried out within $1 \mathrm{~h}$. The analysis was performed using a Cell Cycle Detection Test kit (product code: KGA512; KeyGen).

Western blot analysis. Western blot analysis was conducted as previously described (6). The astrocytes were treated simultaneously with various concentrations of fisetin $(12.5-50 \mu \mathrm{M})$ or $0.1 \%$ DMSO as the control group for $24 \mathrm{~h}$ prior to analysis. The cells were then washed with ice-cold PBS, and total proteins were extracted using the radioimmunoprecipitation assay (RIPA; Sigma-Aldrich) buffer supplemented with phosphatase inhibitors. The concentration of proteins was determined by the bicinchoninic acid (BCA) method with bovine serum albumin (BSA) as a standard using the protein quantification kit (product code, 51254; Sigma-Aldrich). A total of $30 \mu \mathrm{g}$ of protein from each sample was analyzed by SDS/PAGE and transferred by electrophoresis onto polyvinylidene fluoride (PVDF) membranes (Millipore Corp., Billerica, MA, USA). The membranes were blocked for $1 \mathrm{~h}$ at room temperature by using 5\% (w/v) BSA Tris-buffered saline with Tween-20 (TBST) buffer $(20 \mathrm{mM}$ Tris pH 7.5 and $150 \mathrm{mM}$ $\mathrm{NaCl}$ ). The membranes were then incubated with primary antibodies to the following: phosphorylated (p-)focal adhesion kinase (FAK; Tyr 576/577; product code: 3281; diluted 1:1,000), FAK (D2R2E; product code: 13009; diluted 1:1,000), p-paxillin (Tyr118; product code: 2541; diluted 1:1,000), paxillin (product code: 12065; diluted 1:1000), cyclin D1 (product code: 2922; diluted 1:1,000), p-Akt (s473; product code: 4060; diluted 1:2,000), Akt (pan; product code: 4691; diluted 1:1,000), p-p44/42 mitogen-activated protein kinase [MAPK; extracellular signal-regulated kinase (Erk1/2); Thr202/Tyr204; product code: 4370, diluted 1:2,000), p44/42 MAPK (Erk1/2; 137F5; product code: 4695, diluted 1:1,000), p-p38 MAPK (Thr180/Tyr182; D3F9; product code: 4511, diluted 1:1,000), p38 MAPK (D13E1; product code: 8690 , diluted 1:1,000) and glyceraldehyde 3-phosphate dehydrogenase (GAPDH; product code: 5174, diluted 1:1,000. All antibodies were from Cell Signaling Technology, Inc., (Beverly, MA, USA), and were used at $4^{\circ} \mathrm{C}$ overnight. The blots were then incubated with horseradish peroxidase-conjugated anti-rabbit IgG (product code: 7071; all diluted 1:4,500; Cell Signaling Technology, Inc.) for $1 \mathrm{~h}$ at room temperature. Finally, the signals were developed using the enhanced chemiluminescence (ECL) kit (Millipore Corp.), and analyzed using Quantity One software (Bio-Rad Laboratories, Inc., Hercules, CA, USA). Protein levels were expressed as gray values (GVs) relative to the background controls.

Quantitative analysis. The evaluation of the western blot analysis data was carried out using the following steps: i) The GV of the western blot bandings was examined using Image-Pro Plus (IPP) software in the control and experimental group; ii) in each group, the GV of the protein of interest was divided by the $\mathrm{GV}$ of the internal reference; iii) the results of the experimental group from 'step ii' were divided by the results of the control group from 'step ii'; and iv) the final results were presented as the 'fold change'.

Statistical analysis. Data are representative of at least 3 independent experiments. Each experiment was carried out in triplicate. Data were plotted using GraphPad 'PRISM' soft- 
A<smiles>O=c1c(O)c(-c2ccc(O)c(O)c2)oc2cc(O)ccc12</smiles>

C

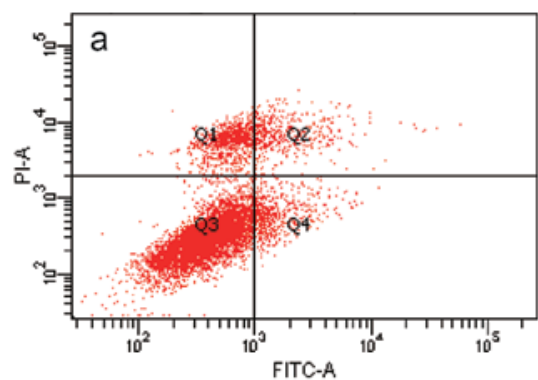

$25(\mu \mathrm{M})$

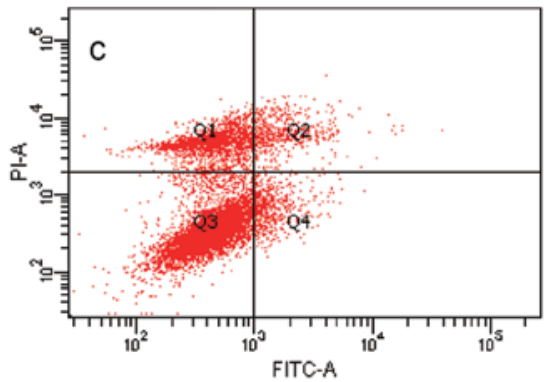

B
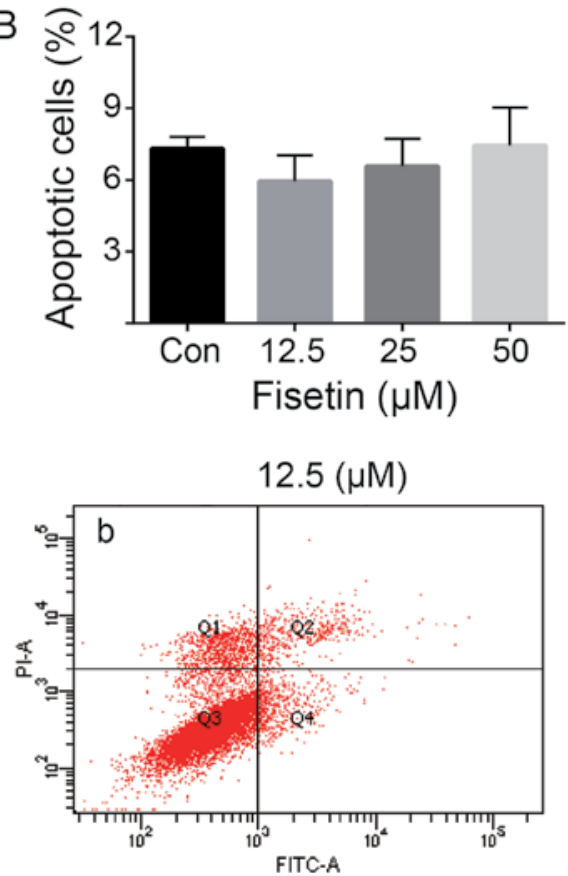

$50(\mu \mathrm{M})$

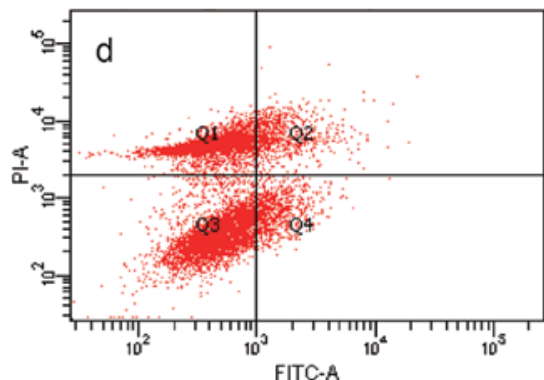

Figure 1. Structure of fisetin and effects on cell migration and apoptosis in primary cultured astrocytes. (A) Chemical structure of fisetin. (B) Quantification of the astrocyte apoptotic rate following treatment with various concentrations of fisetin. (C) Apoptosis of astrocytes treated with or without fisetin for $24 \mathrm{~h}$ as detected by flow cytometry. Fisetin at concentrations ranging from 12.5-50 $\mu \mathrm{M}$ (panels b-d) did not significantly affect cell apoptosis following treatment for $24 \mathrm{~h}$ compared to the control group (panel a).

ware (version 3.0; GraphPad Software, Inc., La Jolla, CA, USA). The significance of the differences was evaluated by one-way ANOVA followed by the Student-Newman-Keuls post hoc test. Error bars represent the standard error of the mean (SEM) and values of $\mathrm{P}<0.05$ and $\mathrm{P}<0.01$ were considered to indicate statistically significant differences.

\section{Results}

Effect of fisetin on astrocyte apoptosis. As shown in Fig. 1B and C, we examined the effect of fisetin on astrocyte cell apoptosis using flow cytometry analysis. Fisetin, at concentrations ranging from $12.5-50 \mu \mathrm{M}$, did not significantly affect cell apoptosis following treatment for $24 \mathrm{~h}$.

Fisetin suppresses astrocyte migration. The effect of fisetin on astrocyte migration was determined using an in vitro scratchwound assay, in which the loss of cell contact induces local chemokine release, which in turn stimulates the cells to close the scratch $(12,13)$. Our results revealed that fisetin significantly decreased the ability of astrocytes to migrate into the empty space (Fig. 2A and B). In the control astrocytes, the wound gap was reduced at $24 \mathrm{~h}$ (Fig. 2A, panel d), while the gap was larger at $24 \mathrm{~h}$ in the fisetin-treated astrocytes (Fig. 2A, panels e and f). Examination of the leading edge of the migrating cells revealed multiple protrusions and extensions in the control astrocytes, but a relatively uniform flat edge in the astrocytes in the fisetin group. To gain further insight into the effects of fisetin on the migration of primary cultured astrocytes, we examined the expression of p-FAK and p-paxillin in vitro. The results of western blot analysis revealed that the expression levels of both p-FAK (Fig. 2C and D) and p-paxillin (Fig. 2C and E) were decreased in the fisetin-treated astrocytes. Taken together, these results indicate that fisetin suppresses astrocyte migration.

Fisetin inhibits astrocyte proliferation and cell cycle progression. Astrocyte proliferation was detected using EdU as a marker of dividing cells (Fig. 3A). The proportion of $\mathrm{EdU}(+)$ cells was markedly reduced in the fisetintreated group (Fig. 3A, panels b-d) compared with the control group (Fig. 3A, panel a), in a dose-dependent manner (Fig. 3A and B). Similarly, fisetin increased the percentage of cells in the G1/G0 phase and decreased the percentage of cells in the $\mathrm{G} 2$ and $\mathrm{S}$ phase (Fig. 3A, panels f-h) 
A

Fisetin $(\mu \mathrm{M})$

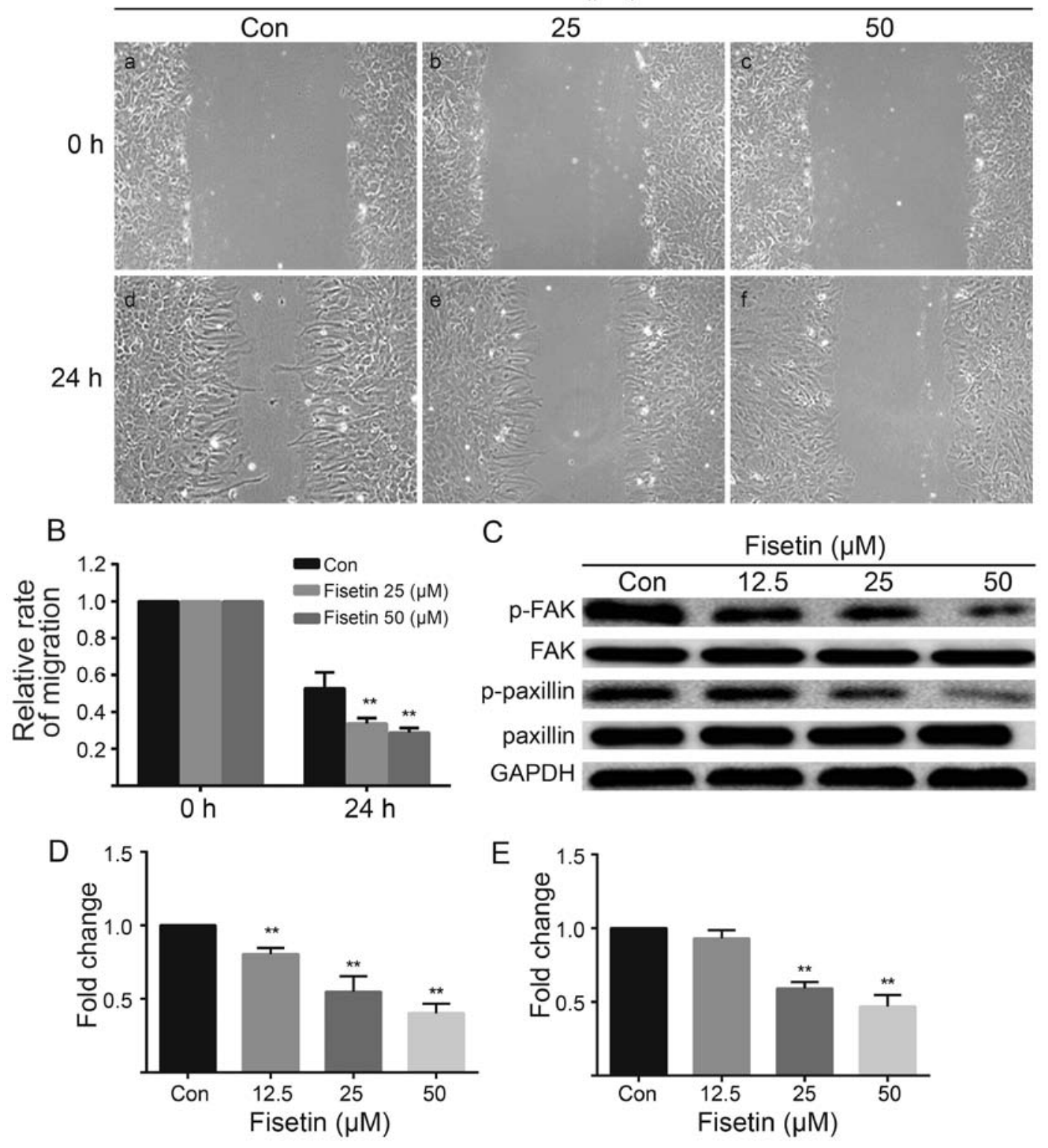

Figure 2. Fisetin suppresses astrocyte migration. (A) Migration profiles showing the inhibition of scratch wound closure by fisetin. (A and B) Fisetin significantly reduced the ability of astrocytes to migrate into the empty space. Control astrocytes reduced the gap at $24 \mathrm{~h}$ (panel d), while the gap was larger at $24 \mathrm{~h}$ in fisetin-treated astrocytes (panels e and f). (B) Quantification of migration rates as a percentage of cell free width. (C) Western blot analysis results showing that both $(\mathrm{C}$ and $\mathrm{D}) \mathrm{p}$-FAK and $(\mathrm{C}$ and $\mathrm{E}) \mathrm{p}$-paxillin expression decreased. (B,D and E) The values are shown as the means $\pm \mathrm{SEM}$ of data from independent experiments. ${ }^{* *} \mathrm{P}<0.01$ vs. control.

compared with the control group (Fig. 3A, panel e), as demonstrated by flow cytometry, and also in a dose-dependent manner (Fig. 3A and C). Accordingly, the results of western blot analysis revealed that fisetin downregulated cyclin D1 protein expression (Fig. 3D and E). Taken together, these data indicated that treatment with fisetin inhibited astrocyte proliferation and arrested the cell cycle at the G1 phase.

Fisetin suppresses the Akt/Erk signaling pathway. Previous studies have demonstrated that fisetin regulates the phosphoinositide 3-kinase (PI3K)/Akt and Erk signaling pathways (14-16). It has also been demonstrated that astrocyte migration is modulated by Rho GTPase/p21-activated kinase (PAK)/Erk-dependent signaling (17). In the present study, the results of western blot analysis revealed that fisetin downregulated the phosphorylation of Akt (Fig. 4A and B) and Erk (Fig. 4A and C) in a dose-dependent manner, but had no effect on total levels of Akt and Erk. Furthermore, fisetin had no effect on the expression of p38 MAPK or p38 phosphorylation levels compared with the control group (Fig. 4A and D).

\section{Discussion}

Astrocytes are ubiquitous glia with finely branching processes that envelope all the cellular components throughout the CNS (5). Under normal physiological conditions, astrocytes provide essential support for neuronal activities, such as maintaining the homeostasis of the extracellular ionic environment and $\mathrm{pH}$, the clearance and release of extracellular glutamate (18), and provisions for metabolic substrates for neurons (19). SCI is 
A Fisetin $(\mu \mathrm{M})$
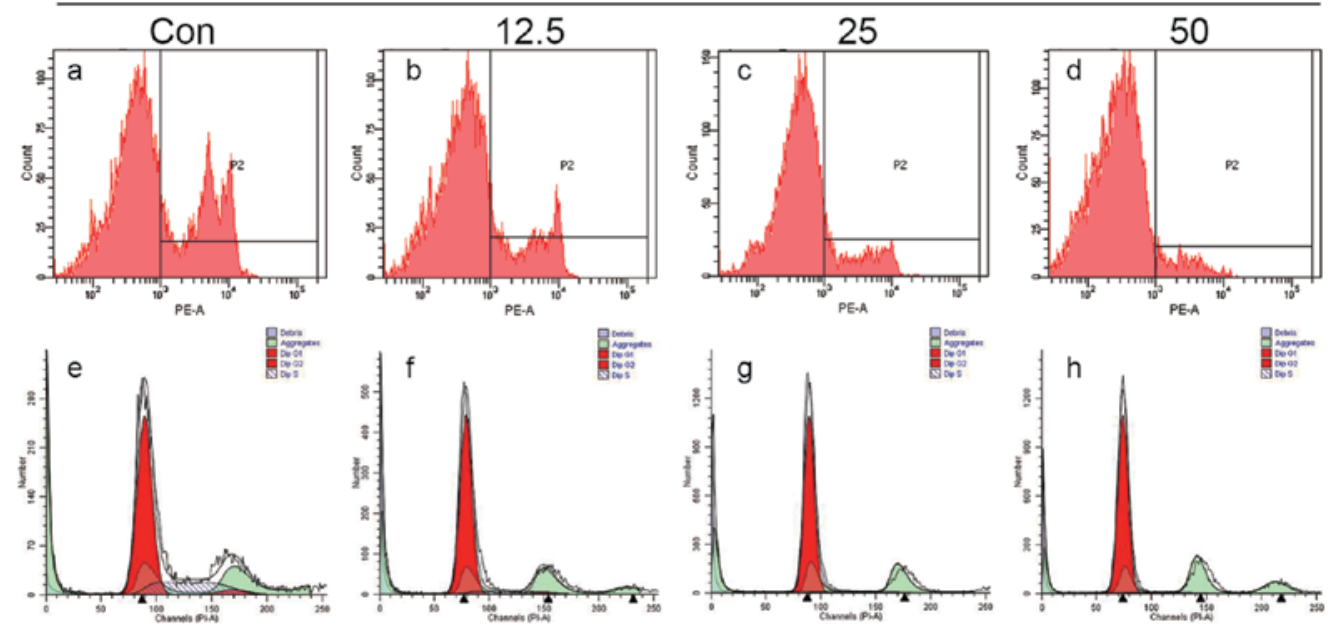

B
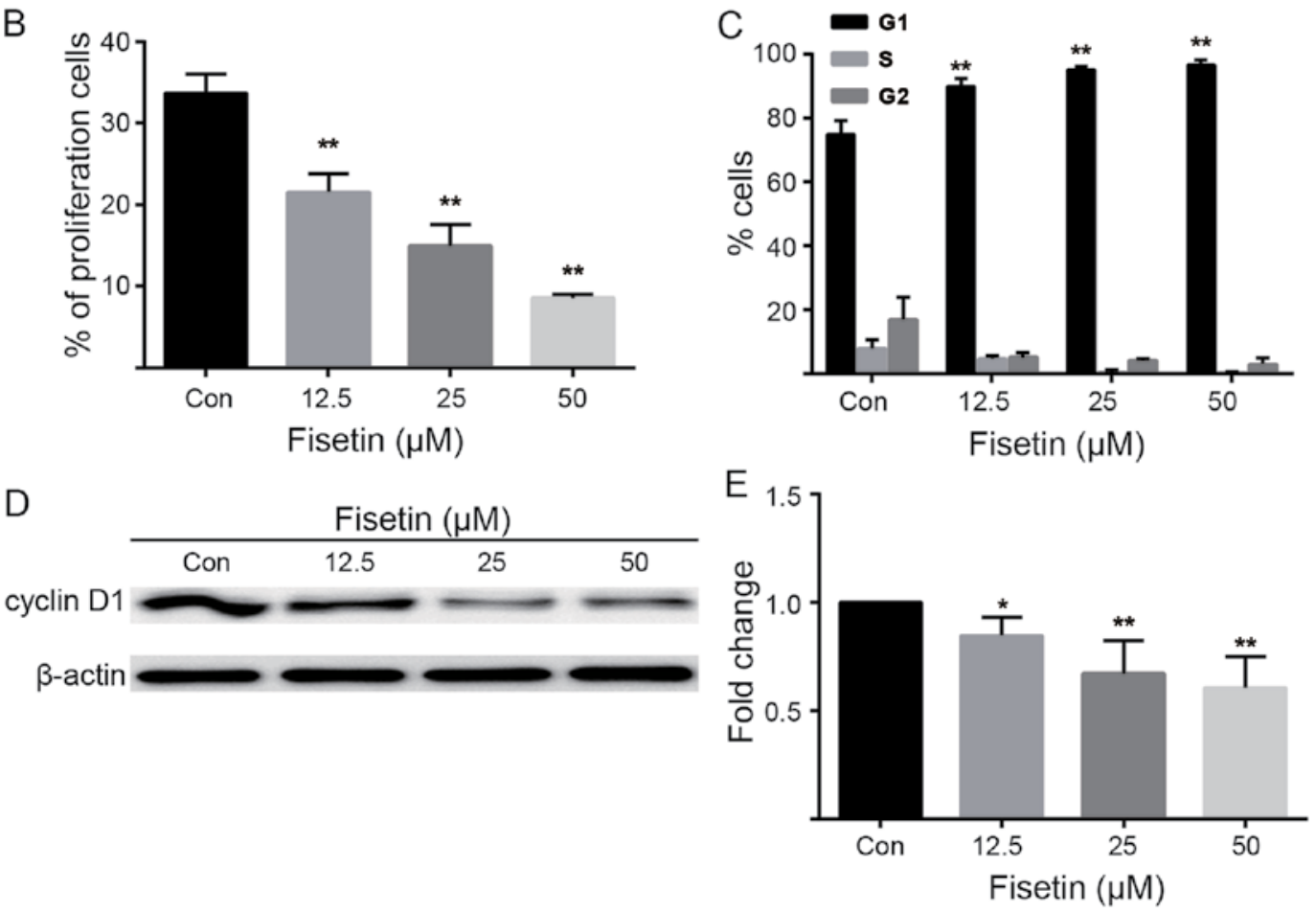

Figure 3. Fisetin inhibits astrocyte proliferation and arrests the cell cycle at the G1 phase in a dose-dependent manner. (A) Astrocyte proliferation was measured using the Click-iT EdU test by flow cytometry. (A and B) The proportion of EdU(+) cells was markedly reduced in the fisetin-treated group (panels b-d) compared with the control group (panel a), in a dose-dependent manner. P2 is the percentage of EdU(+) cells. Fisetin increased the ratio cells in the G1 phase and reduced the ratio of cells in the G2 and S phase (panels e-h). (B) Quantification of astrocyte proliferation ratio with various concentrations of fisetin. (C) Quantification of cell cycle phases in astrocytes treated with various concentrations of fisetin. (D and E) Cyclin D1 expression was downregulated by fisetin in a dose-dependent manner. (B,C and E) The values are shown as the means \pm SEM of data from independent experiments. ${ }^{\prime} \mathrm{P}<0.05,{ }^{* *} \mathrm{P}<0.01$ vs. control.

a common and devastating CNS insult that leads to the destruction of the cord microenvironment, followed by limited neuronal regeneration and poor functional recovery in adult mammals. Following severe SCI, and in response to changes in the local microenvironment, astrocytes, the most abundant glial cells in the CNS, transform into reactive astrocytes (20). Reactive astrogliosis involves cell division, migration, hypertrophy, proliferation and changes in gene expression that contribute to glial scar formation. Compelling evidence suggests that reactive astrogliosis has beneficial effects, including restricting the spread of inflammation, protecting nerves from secondary injuries, and restoring tissue integrity in the acute phase of
CNS insults $(21,22)$. However, studies have also demonstrated the detrimental effects of reactive astrogliosis in terms of interfering with the function of residual neuronal circuits by preventing axonal remyelination and inhibiting axonal regeneration $(23,24)$, thus blocking functional recovery.

Fisetin is a flavonoid and an important natural product derived from many fruits and herbal sources. It has low cytotoxicity and has antitumor effects in various cancer cells, in addition to inducing cell cycle arrest (25). Although previous studies have revealed that fisetin exerts both antioxidant and anti-inflammatory effects in astrocytes $(9,10,26)$, its other activities in astrocytes are unclear. 

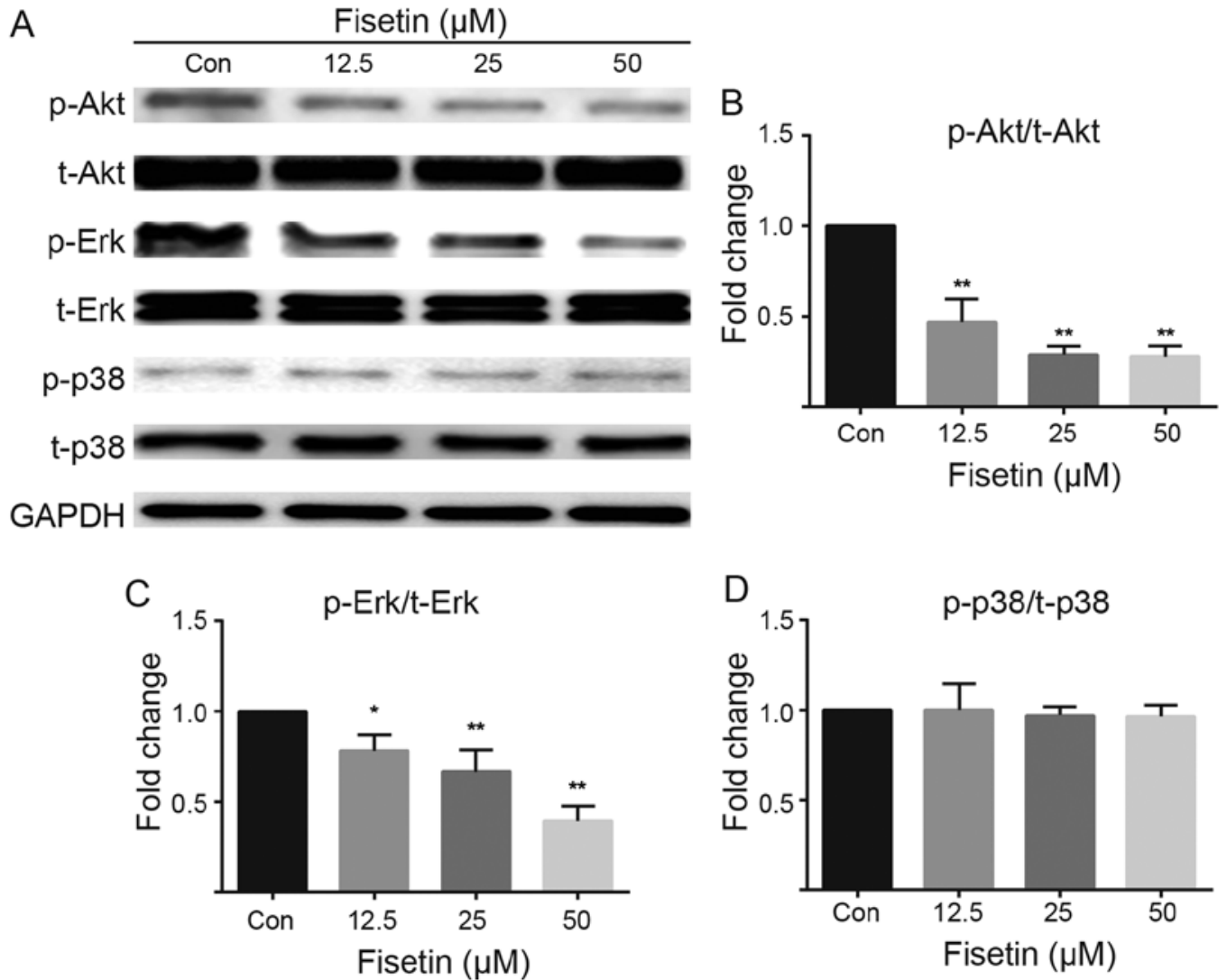

Figure 4. Effect of fisetin on phosphorylated (p-)Akt and total Akt, p-Erk1/2 and total Erk1/2, and p-p38 and total p38 assessed by western blot analysis. (A) Fisetin reduced Akt and Erk1/2 phosphorylation in a dose-dependent manner post-fisetin treatment, but had no effect on p38 phosphorylation. (B,C and D) The values of the quantification of Akt, Erk1/2, and p38 phosphorylation levels are shown as the means \pm SEM of data from independent experiments. ${ }^{*} \mathrm{P}<0.05$, ${ }^{* *} \mathrm{P}<0.01$ by one-way ANOVA followed by the Student-Newman-Keuls post hoc test.

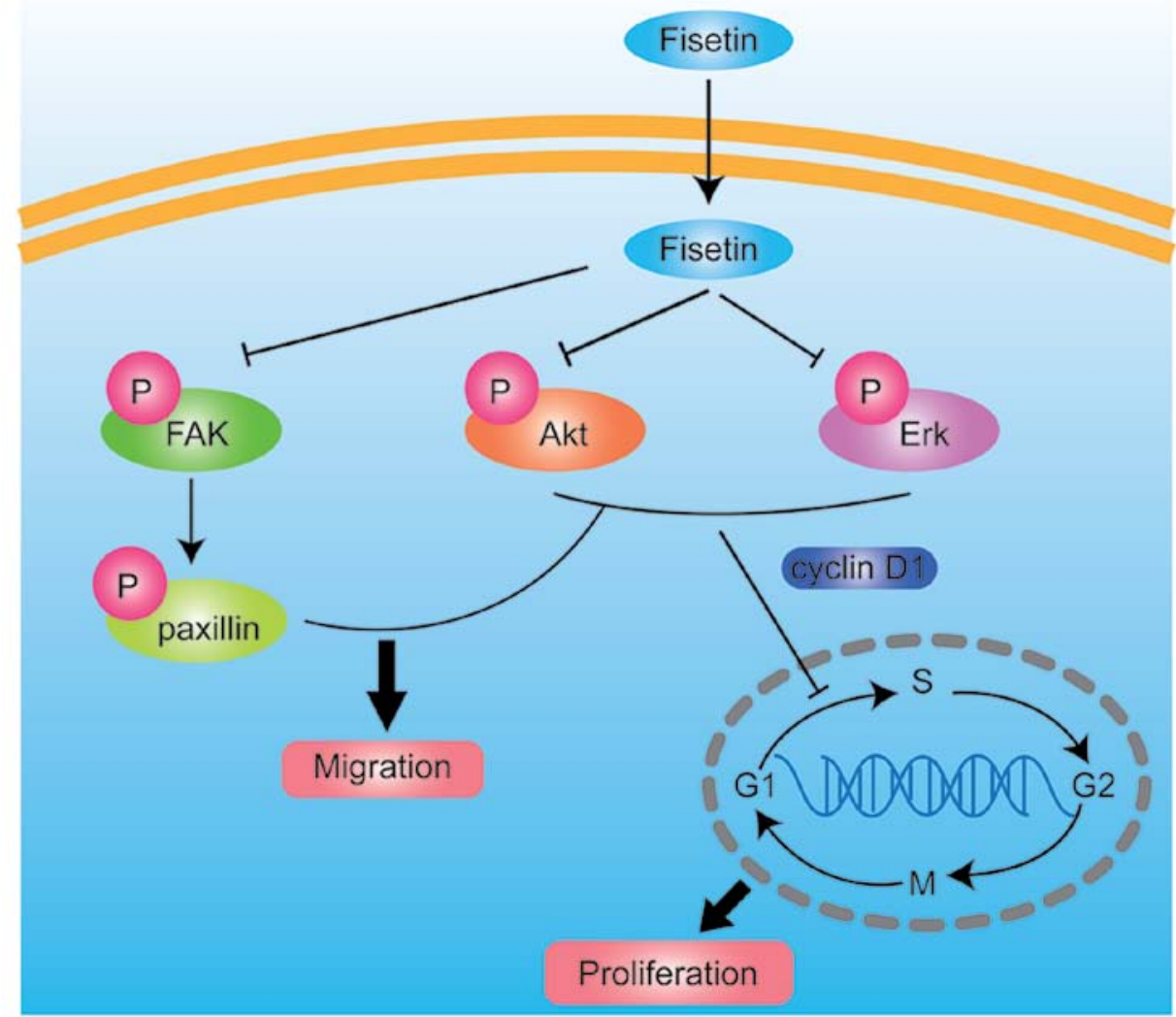

Figure 5. Schematic representation of the possible cellular targets of fisetin action in astrocytes after the scratch wound injury. 
Astrocyte migration and proliferation in $\mathrm{SCI}$ is a fundamental component of glial scarring. In the present study, we demonstrated that fisetin inhibited astrocyte migration in an in vitro scratch-wound model and decreased FAK and paxillin phosphorylation levels in a dose-dependent manner. FAK is a widely expressed cytoplasmic protein tyrosine kinase involved in integrin-mediated signal transduction. It plays an important role in the control of several biological processes, including cell spreading, migration and survival (27). FAK serves as a unique regulator of focal adhesion (FA) assembly and disassembly, processes that are fundamental for efficient directional cell movement $(28,29)$. FAK is a leading edge organizer. Nascent FAs are formed at the cell periphery by integrin and extracellular matrix (ECM) interactions. Paxillin is another important cytoskeletal and scaffolding protein recruited early to nascent FAs at cell fronts, and is necessary for FA turnover (e.g., adhesion disassembly at cell fronts) during cell migration (29,30). Our results indicated that fisetin inhibited healing in the scratch wound model of primary astrocytes and decreased the phosphorylation levels of FAK and paxillin, thus indicating that is plays an essential role in the migratory ability of astrocytes. Furthermore, emerging data indicate that fisetin possesses potent anti-proliferative activity against various cancer cells $(25,31,32)$. Another study reported that fisetin perturbed spindle checkpoint signaling, which may contribute to the anti-proliferative effects of the compound (33). In a previous study, using a cell-based high throughput screen for small molecules that can override chemically-induced mitotic arrest, fisetin was identified as an anti-mitotic compound. It was demonstrated that fisetin rapidly compromised the microtubule drug-induced mitotic block in a proteasome-dependent manner in several human cell lines (33). In this study, we found that fisetin suppressed astrocyte proliferation by inhibiting DNA synthesis, which was shown by EdU, and induced cell cycle arrest in the G1 phase. We also examined the cell cycleassociated protein cyclin D1 by western blot analysis. Cyclin D1 is expressed in the G1 phase and binds to and activates cyclindependent kinase (CDK) 4 and 6 , followed by the activation of the cyclin E-dependent kinase, CDK2.

The MAPK and Akt signaling pathways are crucial for cellular responses including proliferation, differentiation, survival and apoptosis. Accumulating evidence suggests an important role for the MAPK and PI3K/Akt pathways in promoting these biological functions; however, the findings are complex and controversial, and depend on the specific characteristics of the cell type $(34,35)$. A recent study found that the Erk $1 / 2$ signaling pathway modulated the cytoskeleton dynamics of astrocytes in a scratch-wound model (17). Fisetin has been shown to play an important role in regulating the PI3K/Akt and Erk pathways $(14,16,36)$. In the present study, fisetin downregulated the Akt and Erk phosphorylation levels in astrocytes in a dose-dependent manner, but had no effect on p38 expression and phosphorylation. The $\mathrm{p} 38$ protein has been implicated as a key regulator of apoptosis (37), and these results were consistent with the apparent lack of effect of fisetin on apoptosis.

In conclusion, the findings of the present study indicate that fisetin inhibits astrocyte migration, as shown by a scratchwound model and induced cell cycle arrest in the G1 phase. Fisetin inhibited astrocyte proliferation via the PI3K/Akt- and Erk-dependent signaling pathways, and further decreased glial scar formation. The mechanisms involved ares shown in Fig. 5. Fisetin may therefore have potential for use in the treatment of glial scar formation in SCI.

\section{Acknowledgements}

The present study was supported by the Presidential Foundation of Nan Fang Hospital, Southern Medical University, China (grant no. 2013C003)

\section{References}

1. Yang Q, Wang EY, Huang XJ, Qu WS, Zhang L, Xu JZ, Wang W and Tian DS: Blocking epidermal growth factor receptor attenuates reactive astrogliosis through inhibiting cell cycle progression and protects against ischemic brain injury in rats. $\mathbf{J}$ Neurochem 119: 644-653, 2011.

2. Binder DK and Steinhäuser C: Functional changes in astroglial cells in epilepsy. Glia 54: 358-368, 2006.

3. Fuller S, Münch G and Steele M: Activated astrocytes: a therapeutic target in Alzheimer's disease? Expert Rev Neurother 9: 1585-1594, 2009.

4. Holley JE, Gveric D, Newcombe J, Cuzner ML and Gutowski NJ: Astrocyte characterization in the multiple sclerosis glial scar. Neuropathol Appl Neurobiol 29: 434-444, 2003.

5. Sofroniew MV: Reactive astrocytes in neural repair and protection. Neuroscientist 11: 400-407, 2005.

6. Li CY, Li X, Liu SF, Qu WS, Wang W and Tian DS: Inhibition of mTOR pathway restrains astrocyte proliferation, migration and production of inflammatory mediators after oxygen-glucose deprivation and reoxygenation. Neurochem Int 83-84: 9-18, 2015.

7. Wang YF, Zu JN, Li J, Chen C, Xi CY and Yan JL: Curcumin promotes the spinal cord repair via inhibition of glial scar formation and inflammation. Neurosci Lett 560: 51-56, 2014.

8. Adhami VM, Syed DN, Khan N and Mukhtar H: Dietary flavonoid fisetin: a novel dual inhibitor of PI3K/Akt and mTOR for prostate cancer management. Biochem Pharmacol 84: 1277-1281, 2012.

9. Prakash D, Gopinath K and Sudhandiran G: Fisetin enhances behavioral performances and attenuates reactive gliosis and inflammation during aluminum chloride-induced neurotoxicity. Neuromolecular Med 15: 192-208, 2013.

10. Chuang JY, Chang PC, Shen YC, Lin C, Tsai CF, Chen JH, Yeh WL, Wu LH, Lin HY, Liu YS and Lu DY: Regulatory effects of fisetin on microglial activation. Molecules 19: 8820-8839, 2014.

11. Wu BY and Yu AC: Quercetin inhibits c-fos, heat shock protein, and glial fibrillary acidic protein expression in injured astrocytes. J Neurosci Res 62: 730-736, 2000.

12. Yuan Z, Yao F, Hu Z, Sun S and Wu B: Quercetin inhibits the migration and proliferation of astrocytes in wound healing. Neuroreport 26: 387-393, 2015.

13. Cory G: Scratch-wound assay. Methods Mol Biol 769: 25-30, 2011.

14. Chen PY, Ho YR, Wu MJ, Huang SP, Chen PK, Tai MH, Ho CT and Yen JH: Cytoprotective effects of fisetin against hypoxia-induced cell death in PC12 cells. Food Funct 6: 287-296, 2015.

15. Maher P, Dargusch R, Bodai L, Gerard PE, Purcell JM and Marsh JL: ERK activation by the polyphenols fisetin and resveratrol provides neuroprotection in multiple models of Huntington's disease. Hum Mol Genet 20: 261-270, 2011.

16. Chiang HM, Chan SY, Chu Y and Wen KC: Fisetin ameliorated photodamage by suppressing the mitogen-activated protein kinase/matrix metalloproteinase pathway and nuclear factor- $\mathrm{kB}$ pathways. J Agric Food Chem 63: 4551-4560, 2015.

17. Lichtenstein MP, Carriba P, Baltrons MA, Wojciak-Stothard B, Peterson JR, García A and Galea E: Secretase-independent and RhoGTPase/PAK/ERK-dependent regulation of cytoskeleton dynamics in astrocytes by NSAIDs and derivatives. J Alzheimers Dis 22: 1135-1155, 2010.

18. Mazzanti M, Sul JY and Haydon PG: Glutamate on demand: astrocytes as a ready source. Neuroscientist 7: 396-405, 2001.

19. Pellerin L and Magistretti PJ: Neuroenergetics: calling upon astrocytes to satisfy hungry neurons. Neuroscientist 10: 53-62, 2004.

20. Yuan YM and He C: The glial scar in spinal cord injury and repair. Neurosci Bull 29: 421-435, 2013. 
21. Rolls A, Shechter R and Schwartz M: The bright side of the glial scar in CNS repair. Nat Rev Neurosci 10: 235-241, 2009.

22. Silver J and Miller JH: Regeneration beyond the glial scar. Nat Rev Neurosci 5: 146-156, 2004.

23. Sofroniew MV: Molecular dissection of reactive astrogliosis and glial scar formation. Trends Neurosci 32: 638-647, 2009.

24. Wang CX and Shuaib A: Involvement of inflammatory cytokines in central nervous system injury. Prog Neurobiol 67: 161-172, 2002.

25. Chen CM, Hsieh YH, Hwang JM, Jan HJ, Hsieh SC, Lin SH and Lai CY: Fisetin suppresses ADAM9 expression and inhibits invasion of glioma cancer cells through increased phosphorylation of ERK1/2. Tumour Biol 36: 3407-3415, 2014

26. Currais A, Prior M, Dargusch R, Armando A, Ehren J, Schubert D, Quehenberger O and Maher P: Modulation of p25 and inflammatory pathways by fisetin maintains cognitive function in Alzheimer's disease transgenic mice. Aging Cell 13: 379-390, 2014.

27. Schaller MD, Hildebrand JD, Shannon JD, Fox JW, Vines RR and Parsons JT: Autophosphorylation of the focal adhesion kinase, pp125FAK, directs SH2-dependent binding of pp60src. Mol Cell Biol 14: 1680-1688, 1994.

28. Lim Y, Lim ST, Tomar A, Gardel M, Bernard-Trifilo JA Chen XL, Uryu SA, Canete-Soler R, Zhai J, Lin H, et al: PyK2 and FAK connections to p190R ho guanine nucleotide exchange factor regulate RhoA activity, focal adhesion formation, and cell motility. J Cell Biol 180: 187-203, 2008.

29. Hu YL, Lu S, Szeto KW, Sun J, Wang Y, Lasheras JC and Chien S: FAK and paxillin dynamics at focal adhesions in the protrusions of migrating cells. Sci Rep 4: 6024, 2014.
30. Deakin NO and Turner CE: Paxillin comes of age. J Cell Sci 121: 2435-2444, 2008.

31. Syed DN, Suh Y, Afaq F and Mukhtar H: Dietary agents for chemoprevention of prostate cancer. Cancer Lett 265: 167-176, 2008.

32. Khan N, Syed DN, Ahmad N and Mukhtar H: Fisetin: a dietary antioxidant for health promotion. Antioxid Redox Signal 19: 151-162, 2013.

33. Salmela AL, Pouwels J, Varis A, Kukkonen AM, Toivonen P Halonen PK, Perälä M, Kallioniemi O, Gorbsky GJ and Kallio MJ: Dietary flavonoid fisetin induces a forced exit from mitosis by targeting the mitotic spindle checkpoint. Carcinogenesis 30: 1032-1040, 2009.

34. Kim EK and Choi EJ: Pathological roles of MAPK signaling pathways in human diseases. Biochim Biophys Acta 1802: 396-405, 2010

35. Engelman JA: Targeting PI3K signalling in cancer: opportunities, challenges and limitations. Nat Rev Cancer 9: 550-562, 2009.

36. Barbakadze T, Natsvlishvili N and Mikeladze D: Thyroid hormones differentially regulate phosphorylation of ERK and Akt via integrin $\alpha v \beta 3$ receptor in undifferentiated and differentiated PC-12 cells. Cell Biochem Funct 32: 282-286, 2014.

37. Liu Y, Zhang S, Su D, Liu J, Cheng Y, Zou L, Li W and Jiang Y: Inhibiting (pro)renin receptor-mediated p38 MAPK signaling decreases hypoxia/reoxygenation-induced apoptosis in H9c2 cells. Mol Cell Biochem 403: 267-276, 2015. 